\title{
Estudio correlacional de estilos de aprendizaje de estudiantes con modalidad en ciencias naturales
}

\author{
Myriam Fabiola Herrera Cifuentes* \\ Pedro Nel Zapata Castañeda**
}

Artículo recibido: 02-11-2010 y aprobado: 04-05-2012
Resumen: El presente artículo muestra los resultados de una investigación en la que se identificó el estilo de aprendizaje de un grupo de estudiantes de secundaria con base en los modelos de estilos de aprendizaje propuestos por Kolb (1984), Alonso, Gallego y Honey (1994) y Grasha (1996). Una vez identificado el estilo de aprendizaje predominante de cada uno de los sujetos de la población, según los distintos instrumentos propuestos para tal fin por cada uno de los modelos, se examinó si existe alguna correlación entre las distintas categorías de estilos de aprendizaje propuestas por cada uno de los modelos señalados anteriormente.

Los resultados obtenidos no muestran una preferencia de los estudiantes por un único estilo de aprendizaje en cada uno de los modelos estudiados. Se obtuvieron porcentajes altos de preferencias por el estilo activo según el modelo de Alonso, Gallego y Honey, asimilador según el modelo de Kolb y participativo según el modelo de Grasha, sin que los porcentajes obtenidos sean lo suficientemente representativos de la población. Obtenidos los valores de correlación entre cada uno de los estilos de aprendizaje propuestos en cada modelo a través del coeficiente Lambda de Goodman y Kruskal para variables nominales, se encontró que los valores de correlación son prácticamente despreciables, lo que confirmaría, en alguna medida, la independencia de cada uno de los modelos analizados.

Palabras clave: Estilos de aprendizaje, correlación, ciencias naturales.

\section{Correlational study of learning styles of students in science model}

Abstract: This article shows the results of a research study in which a group of secondary school students learning style was identified based on the models of learning styles proposed by Kolb (1984), Alonso, Gallego and Honey (1994) and Grasha (1996). Once identified the predominant learning style in each of the participants based on the proposed instruments for this purpose, the researchers examined whether there was a correlation between the various categories of learning styles proposed by each of the models mentioned above.

The results do not show preference for a unique style of learning in each of the studied models. High percentages of preferences were obtained by the active style according to the model of Alonso, Gallego and Honey, assimilating style, according to the model of Kolb and participatory style based on Grasha's model, but the percentages obtained are not representative enough for this population. After obtaining the values of correlation between each of the learning styles proposed in each model through the Goodman and Kruskal's Lambda coefficient for nominal variables, it was found that the correlation values are virtually negligible, results that would confirm, to some extent, the independence of each of the analyzed models.

Key Words: Learning styles, correlation, natural sciences.

\footnotetext{
* Magister en Docencia de la Química. myriamfabiola@hotmail.com

* Doctor en Educación. Profesor Departamento de Química. Universidad Pedagógica Nacional. pzapata@pedagogica.edu.co
} 


\section{Introducción}

Cuando se habla de 'estilo de aprendizaje', se hace referencia al hecho que cada persona utiliza su propio método o conjunto de estrategias para aprender. Aunque las estrategias concretas que utilizan las personas varían según lo que se quiera aprender, cada persona tiende a desarrollar unas preferencias globales. Esas preferencias o tendencias a utilizar más unas determinadas maneras de aprender que otras constituyen el llamado "estilo de aprendizaje" (Lozano, 2006).

Los estilos de aprendizaje son un aspecto importante para estudiar desde el punto de vista educativo, puesto que permiten conocer las tendencias de cómo los estudiantes se desempeñan en el aula. Teniendo en cuenta dicha aproximación, se pueden diseñar y mejorar las estrategias de enseñanza para conseguir un proceso educativo más efectivo.

Para llevar a la práctica la teoría de los estilos de aprendizaje es imprescindible hacer un diagnóstico adecuado. Por eso la mayoría de autores que han trabajado en estilos de aprendizaje, han propuesto diversos instrumentos que posibilitan este diagnóstico. Sin embargo, pocas investigaciones han examinado si existen relaciones entre las distintas categorías de estilos propuestas por cada uno de los modelos de estilos de aprendizaje. De acuerdo con lo anterior, en esta investigación se examinó si existe relación entre los distintos modelos de estilos de aprendizaje propuestos por Kolb (1984), Alonso, Gallego y Honey (1994) y Grasha (1996). Para evaluar la relación entre cada uno de estos modelos se empleó el coeficiente de correlación Lambda para variables nominales.

\section{Antecedentes}

A nivel internacional y nacional se encuentra una cantidad significativa de trabajos, en los cuales se aplican las técnicas de reconocimiento de estilos de aprendizaje a distintos niveles, especialmente en el ámbito universitario. Cada trabajo presenta finalidades diferentes, se aplica en áreas específicas y su finalidad principal ha sido mejorar el desempeño de los estudiantes en dichas áreas.

Por otra parte, también se han llevado a cabo estudios que han relacionado, por ejemplo, los estilos cognitivos de los estudiantes con los estilos cognitivos de los profesores, (Ramírez, 1973, citado por McKenna, 1990); los estilos cognitivos y de enseñanza de los profesores (Riding, 2002; Cárdenas, Oviedo y Zapata, 2009); el estilo cognitivo DIC y las estrategias de aprendizaje de los estudiantes (Frank, 1983); los estilos de aprendizaje y la inteligencia ( Clark y Kreig, 1988); el estilo cognitivo DIC y el estilo de aprendizaje (Witkin y Goodenough, 1981; Salkind y Wright, 1977).

En Colombia se encuentran algunos estudios que han examinado los estilos de aprendizaje y su relación con otras variables como el uso de estrategias pedagógicas (Correa, 2006); los estilos de aprendizaje de estudiantes de educación secundaria (Oviedo, Cárdenas, Zapata, Rendón, Rojas y Figueroa, 2010); y los estilos de aprendizaje y su relación con el rendimiento académico (Prado, 2005).

Luego de revisados estos antecedentes, es de notar que, en el caso colombiano, no se encuentran trabajos que se encarguen de caracterizar los estilos de aprendizaje de los estudiantes de ciencias naturales en la educación media. 
Las investigaciones encontradas se han realizado con estudiantes universitarios, donde está bien delimitada la población hacia instituciones y carreras particulares. Por otra parte, la mayoría de estos trabajos muestra cómo cada investigador se matricula con autores diferentes, pero no se evidencia trabajo alguno que se encargue de comparar los resultados que arrojan diferentes instrumentos. En este sentido, Alonso y Gallego (2003) afirman, por ejemplo, que las investigaciones europeas y australianas sobre estilos de aprendizaje no han sido generalmente analizadas y contrastadas, de forma que las panorámicas y estudios comparativos quedan, con frecuencia, incompletos.

\section{Marco de referencia}

Según Camargo y Hederich (2007) "la noción general de estilo no tiene su origen en el entorno educativo. Esta proviene principalmente de las artes y se refiere al conjunto de características que definen una tendencia estética identificable y distintiva” (p. 32). Según estos mismos autores "el término estilo comienza a utilizarse en psicología hacia la década de 1950 para referirse a ciertos rasgos diferenciadores o individualizadotes en la caracterización de una persona" (p. 32).

Según Lozano (2006), en la literatura existente, al referirse a los estilos (cognitivos, de personalidad, de aprendizaje, enseñanza e intelectuales), varios autores hacen alusión a preferencias o gustos (Stenrberg, 1997; Hirsh y Kummerow, 1990; Dunn y Dunn, 1978), patrones conductuales que pueden o no ser observables y estrategias de aprendizaje ( Riding y Rayner, 1999; Guild y Garger,
1985) y a habilidades y fortalezas (Gardner, 1983).

Según Lozano (2006), algunos autores como Guild y Garger (1985) postulan algunos supuestos básicos acerca de los estilos entre los que se destacan:

a. Cada persona tiene su propio estilo.

b. Los estilos son neutrales. No hay estilos mejores o peores.

c. Los estilos son estables, pero algunos patrones de conducta pueden variar dependiendo de la situación.

d. Los estilos no son absolutos.

e. Los estilos en sí mismos no manifiestan competencia.

Adicionalmente, autores como Sternberg (1997) han señalado algunos principios acerca de los estilos, entre los que se destacan:

a. Los estilos son preferencias en el uso de habilidades, pero no son habilidades en sí mismas.

b. Una relación entre los estilos y las habilidades genera una sinergia más importante que la simple suma de las partes.

c. La gente tiene perfiles o patrones de estilos, no un solo estilo.

d. Los estilos son variables de acuerdo con las tareas y las situaciones.

e. La gente difiere en la fuerza de sus preferencias.

f. Las personas difieren en su flexibilidad estilística. 
g. Los estilos son socializados.

h. Los estilos pueden variar a lo largo de la vida.

i. Los estilos pueden ser mesurables.

j. Los estilos pueden enseñarse.

James y Gardner (1995) definen los estilos de aprendizaje como "...la manera compleja en la cual y bajo ciertas condiciones, los estudiantes aprenden más eficientemente y perciben más efectivamente, procesan, almacenan y evocan lo que están intentando aprender".

El término "estilo de aprendizaje" se refiere al hecho de que cada persona utiliza su propio método o estrategia a la hora de aprender. Aunque las estrategias varían según lo que se quiera aprender, cada uno tiende a desarrollar ciertas preferencias o tendencias globales, tendencias que definen un estilo de aprendizaje. Se habla de una tendencia general, puesto que, por ejemplo, alguien que casi siempre es auditivo puede en ciertos casos utilizar estrategias visuales.

Revilla (1998) destaca algunas características de los estilos de aprendizaje: son relativamente estables, aunque pueden cambiar; pueden ser diferentes en situaciones diferentes; son susceptibles de mejorarse y cuando a los alumnos se les enseña según su propio estilo de aprendizaje, aprenden con más efectividad.

Lozano (2006) presenta los principios teóricos en que se fundamenta el modelo de estilos de aprendizaje de Dunn y Dunn (1978), entre los que se destacan los siguientes: "1. El estilo de aprendizaje es un conjunto biológico y del desarrollo de características personales que hacen que ambientes, métodos y recursos instruccionales idénticos sean eficaces para algunos alumnos e ineficaces para otros. 2. La mayoría de la gente tiene ciertas preferencias de estilos de aprendizaje, pero estas preferencias difieren de manera significativa. 3. Los profesores pueden aprender a usar los estilos de aprendizaje como una base sólida en la preparación de sus programas académicos. 4. Cuanto menor sea el éxito académico en una persona, mayor es la importancia de acomodar sus preferencias de estilos de aprendizaje a experiencias de aprendizaje adecuadas".

Sin embargo, es importante no utilizar los estilos de aprendizaje como una herramienta para clasificar a los alumnos en categorías cerradas, ya que la manera de aprender evoluciona y cambia constantemente. Los estilos son variables de acuerdo con las tareas y las situaciones. Según Guild y Garger (1985) los estilos no son absolutos. Las personas pueden adaptarse a las situaciones y a las tareas según determinados factores, como el estado de ánimo, la motivación, la disposición, la necesidad, etc." (Lozano, 2006).

Otros autores, por último, sugieren hablar de "preferencias de estilos de aprendizaje" más que de "estilos de aprendizaje”. Para Woolfolk (1995) las preferencias son una clasificación más precisa, y se definen como las maneras preferidas de estudiar y aprender tales como utilizar imágenes en vez de texto, trabajar solo o con otras personas, aprender en situaciones estructuradas o no estructuradas y demás condiciones pertinentes como un ambiente con o sin música, el tipo de silla utilizado, etc. La 
preferencia de un estilo particular tal vez no siempre garantice que la utilización de ese estilo será efectiva. De allí que en estos casos ciertos alumnos pueden beneficiarse desarrollando nuevas formas de aprender.

Las teorías sobre estilos de aprendizaje han tomado un fuerte auge en los últimos 30 años. En realidad existen diversas propuestas para clasificar los distintos estilos de aprendizaje de las personas, atendiendo a diversidad de criterios y de objetivos. A continuación, se presentan las características principales de los modelos de estilos de aprendizaje que se tuvieron en cuenta para el desarrollo de la presente investigación, los cuales, en algunos casos, atienden a factores de personalidad y, en otros, a factores como el comportamiento, la cognición y el pensamiento.

\section{El modelo de Kolb}

Según Kolb (1984), el aprendizaje es el proceso de adquirir y recordar ideas y conceptos. Según este autor, para ser eficaz, el aprendiz necesita cuatro clases diferentes de capacidades: a) experiencia concreta, b) observación reflexiva, c) conceptualización abstracta y d) experimentación activa. Con base en el desarrollo del modelo propuesto, Kolb identificó cuatro tipos predominantes de estilos de aprendizaje: convergente, divergente, asimilador y acomodador. Kolb creó un instrumento al que denominó inventario de estilos de aprendizaje (LSI) (Ver anexo 1), que es un cuestionario compuesto por doce series de palabras que es preciso ordenar por preferencia. Cada palabra representa uno de los estilos de aprendizaje propuestos.

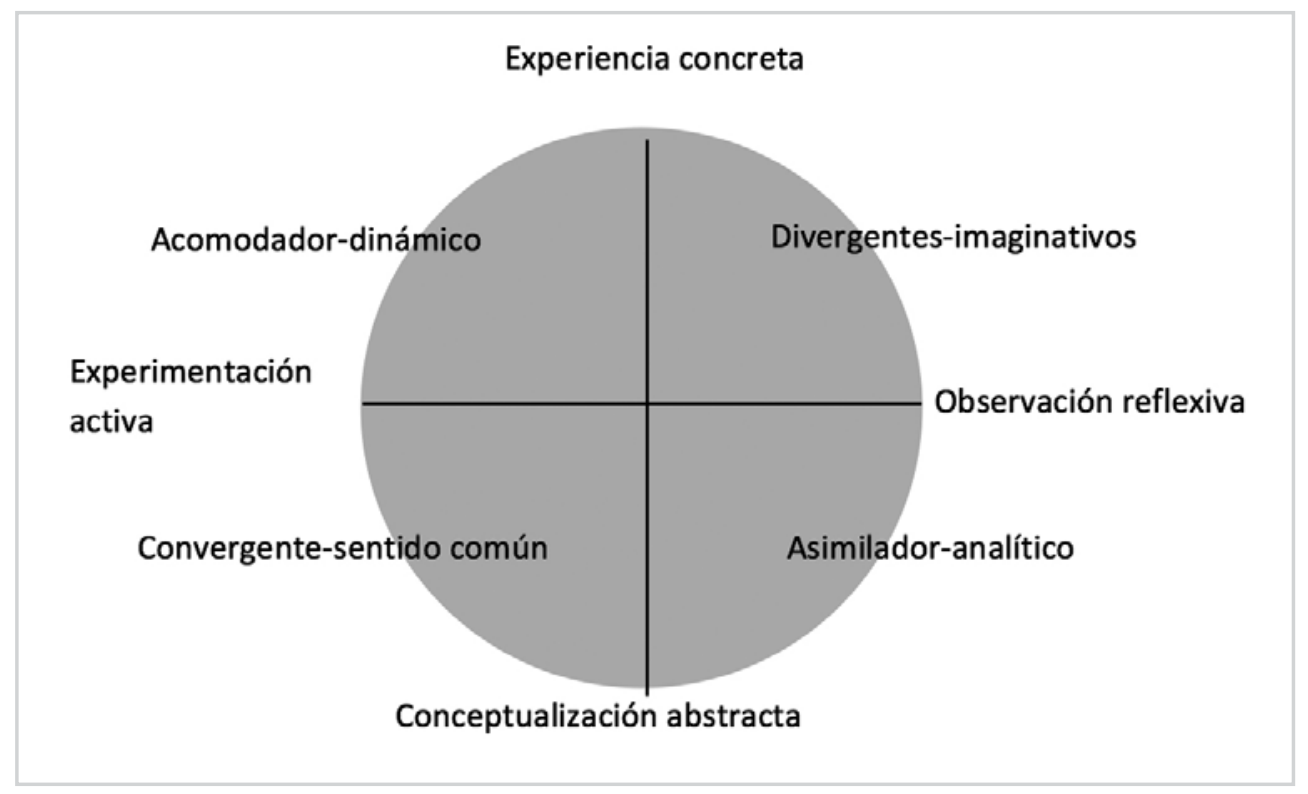

Figura 1. Modelos de aprendizaje de Kolb. Adaptación 
A continuación se describen algunas características de los estilos de aprendizaje propuestos por Kolb

\begin{tabular}{|c|c|c|c|}
\hline Convergente & Divergente & Asimilador & Acomodador \\
\hline $\begin{array}{l}\text { Pragmático } \\
\text { Racional } \\
\text { Analítico } \\
\text { Gusta de la } \\
\text { experimentación } \\
\text { Es poco empático } \\
\text { Hermético } \\
\text { Buen líder } \\
\text { Son deductivos }\end{array}$ & $\begin{array}{l}\text { Sociable } \\
\text { Sintetiza bien } \\
\text { Orientado a las personas } \\
\text { Empático } \\
\text { Muy imaginativo } \\
\text { Emocional } \\
\text { Flexible } \\
\text { Intuitivo }\end{array}$ & $\begin{array}{l}\text { Poco sociable } \\
\text { Genera modelos } \\
\text { Reflexivo } \\
\text { pensador } \\
\text { Disfruta la teoría } \\
\text { Poco empático } \\
\text { Planificador } \\
\text { Poco sensible }\end{array}$ & $\begin{array}{l}\text { Sociable } \\
\text { Acepta retos } \\
\text { Impulsivo } \\
\text { Orientado a la acción } \\
\text { Poca habilidad analítica } \\
\text { Empático } \\
\text { Flexible }\end{array}$ \\
\hline
\end{tabular}

Cuadro 1. Características de los estilos de aprendizaje de Kolb

\section{El modelo de Alonso, Gallego y Honey}

Según Alonso, Gallego y Honey (1994), quienes a su vez usan como soporte las teorías e inventarios de estilos de aprendizaje de Kolb (1984), los estilos de aprendizaje serán la interiorización, por parte de cada sujeto, de una etapa determinada de su ciclo de aprendizaje. Para estos autores los estilos de aprendizaje son cuatro, que a su vez corresponden a las cuatro fases del proceso cíclico de aprendizaje: activo, teórico, reflexivo y pragmático. Estos investigadores adaptaron el cuestionario LSQ de estilos de aprendizaje al ámbito académico y al idioma español. Alonso denominó a este cuestionario test CHAEA (Cuestionario Honey-Alonso de estilos de aprendizaje) (Ver anexo 2).

El CHAEA cuenta con 80 ítems, cada ítem se responde con un signo (+) si se está de acuerdo y con un (-) si se está en desacuerdo. CHAEA está basado en teorías del aprendizaje de tipo cognitivo y coincide con el inventario de Kolb (1984) al atender a dos dimensiones principales durante el proceso de aprendizaje: por un lado, el cómo percibimos la nueva información y, por otro, cómo procesamos lo que percibimos. Según Alonso, Gallego y Honey (1994), en función de la primera dimensión, el proceso de aprendizaje se presenta bajo el indicador "activo" en un extremo y "teórico" en el otro. En el segundo caso, nos encontramos ante otro indicador en cuyos extremos se encuentran el "reflexivo" y el "pragmático". Algunas características de estos estilos se describen a continuación:

Estilo activo: las personas que tienen predominancia en estilos activos se implican plenamente sin prejuicio en nuevas experiencias. Son de mente abierta, nada escépticos y realizan con entusiasmo las tareas nuevas. Son personas que se desarrollan en el presente y les fascina vivir nuevas experiencias. Piensan que por lo menos una vez hay que intentarlo todo. Al terminar una actividad entran rápidamente en otra, les aburre los plazos largos, son personas leales al grupo, se involucran en los asuntos de los demás y centran a su alrededor todas sus actividades. 
Estilo teórico: adaptan e integran las observaciones dentro de las teorías lógicas y complejas. Enfocan los problemas de forma vertical, escalonada, por etapas lógicas. Tienden a ser perfeccionistas. Integran los hechos en teorías coherentes. Les gusta analizar y sintetizar. Son profundos en sus sistemas de pensamiento. A la hora de establecer principios, teorías y modelos buscan la racionalidad y la objetividad, huyen de lo subjetivo y lo ambiguo.

Estilo reflexivo: les gusta considerar las experiencias y observarlas desde diferentes perspectivas. Son personas prudentes que gustan considerar todas las alternativas posibles antes de realizar un movimiento. Disfrutan observando la actuación de los demás, escuchan a los demás y no intervienen hasta que se han adueñado de la situación. Crean a su alrededor un aire ligeramente distante y condescendiente.

Estilo pragmático: el punto fuerte de las personas con predominancia del estilo pragmático es la aplicación práctica de ideas. Descubren el aspecto positivo de las nuevas ideas y aprovechan la primera oportunidad para experimentarlas. Les gusta actuar rápidamente y con seguridad en aquellas ideas y proyectos que le atraen. Tienden a ser impacientes cuando hay personas que teorizan. "Pisan la tierra" cuando hay que tomar decisiones o resolver un problema. Su filosofía es "siempre se puede hacer mejor", "si funciona es bueno".

\section{El modelo Grasha}

Otro de los modelos de estilos de aprendizaje que ha llamado especialmente la atención en los últimos años fue el propuesto por Grasha (1996). Este modelo se basa en la observación de los patrones de conducta relacionados con las preferencias de los alumnos a la hora de interactuar con sus compañeros y con sus profesores en el salón de clase.

Grasha (1996) propuso seis estilos de aprendizaje basados en tres dimensiones de carácter bipolar, a saber: las actitudes del estudiante hacia el aprendizaje (participativo versus elusivo), las perspectivas sobre los compañeros y los maestros (competitivo versus colaborativo) y las reacciones a los procedimientos didácticos dentro del salón de clases (dependiente versus independiente). Según este autor, a pesar de que estos estilos en cada dimensión sean bipolares, no significa que no puedan complementarse, ya que solo representan extremos, entre los cuales se pueden conformar distintos tipos de perfiles. Grasha construye un instrumento que consiste en un cuestionario de 60 ítems (Ver anexo 3). El cuestionario trata de averiguar las actitudes de los estudiantes acerca de los cursos de nivel medio superior y superior y en consecuencia de sus estilos de aprendizaje. Algunas características de los estilos propuestos por este autor se describen a continuación: 


\begin{tabular}{l|l}
$\begin{array}{l}\text { Participativo } \\
\begin{array}{l}\text { Son buenos elementos en clase, disfrutan la sesión y } \\
\text { procuran estar pendientes la mayor parte del tiempo. } \\
\text { Tienen mucha disposición para el trabajo escolar. }\end{array}\end{array}$ & $\begin{array}{l}\text { Elusivo } \\
\text { No manifiestan entusiasmo en clase. No participan y } \\
\text { se mantienen aislados. Son apáticos y desinteresados } \\
\text { en las actividades escolares. No les gusta estar mucho } \\
\text { tiempo en el aula. }\end{array}$ \\
\hline $\begin{array}{l}\text { Competitivo } \\
\text { Estudian para demostrar su supremacía en términos } \\
\text { de aprovechamiento o calificación a los demás. }\end{array}$ & $\begin{array}{l}\text { Les gusta aprender compartiendo ideas y talentos. } \\
\text { Gustan de trabajar con sus compañeros y con sus } \\
\text { pes gusta ser el centro de atención y recibir } \\
\text { reconocimiento de sus logros. }\end{array}$ \\
\hline $\begin{array}{l}\text { Dependiente } \\
\begin{array}{l}\text { Manifiestan poca curiosidad intelectual y aprenden } \\
\text { solo lo que tienen que aprender.Visualizan a los } \\
\text { profesores y a sus compañeros como figuras de guía } \\
\text { ylo autoridad para realizar sus actividades. }\end{array}\end{array}$ & $\begin{array}{l}\text { Independiente } \\
\text { Les gusta pensar en sí mismos. Son autónomos } \\
\text { importante y lo que no lo es, y gustan de trabajar de }\end{array}$ \\
\hline manera solitaria. Evitan el trabajo en equipo.
\end{tabular}

Cuadro 2. Características de los estilos de aprendizaje de Grasha (1996)

\section{Metodología}

La investigación adelantada se fundamenta, según la clasificación propuesta por Sierra (1989), en un diseño de tipo seccional en el que a partir de la observación de la población mediante la técnica de cuestionario, se examinó la relación entre los estilos de aprendizaje de cada sujeto de la población según los modelos de estilos señalados anteriormente.

La aplicación de los instrumentos se llevó a cabo en la ciudad de Bogotá, en febrero del año 2010. Se escogió una muestra por conveniencia de 208 estudiantes pertenecientes a los colegios CEDID Guillermo Cano Isaza, Gimnasio La Fontana, colegio Madre Matilde y el IED Atanasio Girardot. Los estudiantes que hicieron parte del estudio pertenecían a los grados $10^{\circ}$ y $11^{\circ}$, correspondiente al ciclo $\mathrm{V}$ de educación media con modalidad técnica de educación o énfasis en ciencias naturales. Las edades de estos estudiantes oscilaron entre los 14 y 19 años.

\section{Resultados y discusión}

A continuación se presentan los resultados obtenidos en cada uno de los test de estilos de aprendizaje empleados en la investigación.

\begin{tabular}{|c|c|c|}
\hline Estilo de aprendizaje & No. de Estd. & $\%$ \\
\hline Divergente & 43 & 20,67 \\
\hline Convergente & 32 & 15,38 \\
\hline Asimilador & 68 & 32,69 \\
\hline Adaptador & 65 & 31,25 \\
\hline
\end{tabular}

\section{Gráfica 1. Resultados test de Kolb}

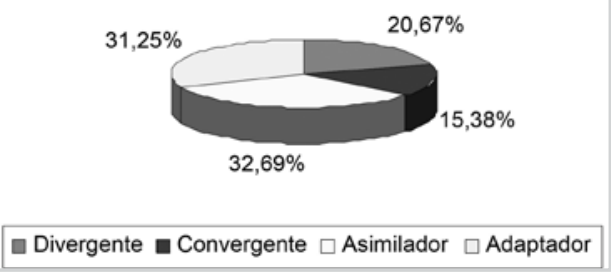

Tabla 1. Distribución de estudiantes en número y porcentaje según estilos de aprendizaje de Kolb. Test LSI 
En los resultados obtenidos para el test de Kolb no se encuentra un estilo que sea predominante sobre los demás, más bien se obtuvieron porcentajes similares para los estilos asimilador con un $32,69 \%$ y adaptador con $31,25 \%$.

\begin{tabular}{|c|c|c|}
\hline Estilo de aprendizaje & No. de Estd. & $\%$ \\
\hline Activo & 84 & 32,30 \\
\hline Reflexivo & 82 & 31,53 \\
\hline Teórico & 51 & 19,61 \\
\hline Pragmático & 43 & 16,53 \\
\hline
\end{tabular}

\section{Grafica 2. Resultados Test CHAEA}

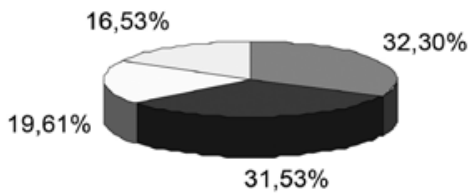

Activo $\square$ Reflexivo $\square$ Teórico $\square$ Pragmático

Tabla 2. Distribución de estudiantes en número y porcentaje según estilos de aprendizaje de Alonso, Gallego y Honey. Test CHAEA

Para el test CHAEA se encontraron dos estilos con los más altos porcentajes, aunque sus valores tan cercanos indican que no hay una tendencia bien definida: el estilo activo obtuvo un 32,3\% y el reflexivo un $31.5 \%$. Las personas con estilo activo se caracterizan por ser estudiantes que se implican plenamente sin prejuicio en nuevas experiencias. Son de mente abierta, nada escépticos y realizan con entusiasmo las tareas nuevas. Son personas que se desarrollan en el presente y les fascina vivir nuevas experiencias. Piensan que por lo menos una vez hay que intentarlo todo. Al ter- minar una actividad entran rápidamente en otra, les aburre los plazos largos, son personas leales al grupo, se involucran en los asuntos de los demás y centran a su alrededor todas sus actividades.

\begin{tabular}{|l|c|c|}
\hline Estilo de aprendizaje & No. de Estd. & $\%$ \\
\hline Independiente & 42 & 17,14 \\
\hline Dependiente & 46 & 18,77 \\
\hline Evasivo & 10 & 4,08 \\
\hline Colaborativo & 57 & 23,26 \\
\hline Participativo & 66 & 26,93 \\
\hline Competitivo & 24 & 9,79 \\
\hline
\end{tabular}

Grafica 3. Resultados Test Grasha Riechmann

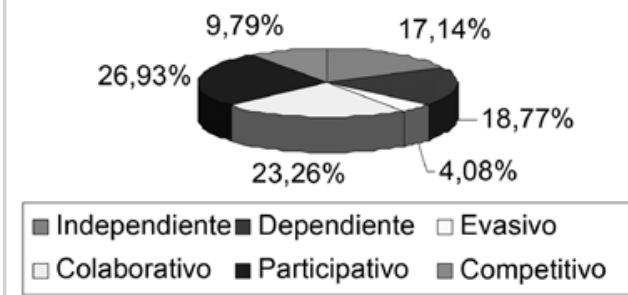

Tabla 3. Distribución de estudiantes en número y porcentaje según estilos de aprendizaje de Grasha

Finalmente, para el test de Grasha los estilos con mayor porcentaje fueron el colaborativo con un 23,26\% y el participativo con $26.93 \%$. Las personas con estilo participativo se caracterizan por resultar buenos elementos en clase puesto que disfrutan de las sesiones y procuran estar pendientes de la misma la mayor parte del tiempo. Tienen mucha disposición para el trabajo escolar.

Como es notorio para las tres propuestas de estilos de aprendizaje, no existen tendencias definidas, ni predominios marcados de unos estilos sobre otros. Así pues, aunque en principio 
208 estudiantes participaron en la investigación, se presentaron casos en los que algunos estudiantes obtuvieron las puntuaciones más altas en dos o más estilos, por lo que se tuvo en cuenta para los cálculos posteriores de correlación cada uno de los estilos en los que los sujetos puntuaron igual. Estos resultados confirman algunos planteamientos señalados en el marco referencial, como es el hecho que los estudiantes no tie- nen un estilo absoluto, sino que tienen perfiles o patrones de estilo.

\section{Correlación entre los modelos de estilos de aprendizaje}

A continuación se presentan las tablas que sirvieron para establecer la correlación entre los distintos modelos de estilos de aprendizaje.

\begin{tabular}{|l|cccc|c|}
\hline Estilos de aprendizaje & Kolb & & & & \\
Grasha & Divergente & Convergente & Asimilador & Adaptador & Total \\
Independiente & 4 & 0 & 10 & 6 & 20 \\
Evasivo & 4 & 0 & 4 & 2 & 10 \\
Colaborador & 15 & 24 & 20 & 18 & 77 \\
Dependiente & 11 & 16 & 11 & 18 & 56 \\
Competitivo & 6 & 5 & 7 & 10 & 28 \\
Participativo & 10 & 13 & 24 & 21 & 68 \\
Total & 50 & 58 & 76 & 75 & 259 \\
\hline
\end{tabular}

Tabla 4. No. de estudiantes según estilos de aprendizaje de Kolb y Grasha

\begin{tabular}{|l|cccc|c|}
\hline Estilos de aprendizaje & CHAEA & & & & \\
Grasha & Activo & Reflexivo & Teórico & Pragmático & Total \\
Independiente & 11 & 7 & 3 & 5 & 26 \\
Evasivo & 5 & 1 & 3 & 3 & 12 \\
Colaborador & 26 & 26 & 13 & 13 & 78 \\
Dependiente & 22 & 22 & 14 & 14 & 72 \\
Competitivo & 13 & 9 & 5 & 9 & 36 \\
Participativo & 23 & 27 & 21 & 15 & 86 \\
Total & 100 & 92 & 59 & 59 & 310 \\
\hline
\end{tabular}

Tabla 5. No. de estudiantes según estilos de aprendizaje CHAEA y Grasha 


\begin{tabular}{|l|llll|l|}
\hline Estilos de aprendizaje & CHAEA & & & \\
Kolb & Activo & Reflexivo & Teórico & Pragmático & Total \\
Divergente & 12 & 18 & 12 & 10 & 52 \\
Convergente & 14 & 24 & 6 & 6 & 50 \\
Asimilador & 24 & 33 & 19 & 18 & 94 \\
Adaptador & 30 & 19 & 15 & 15 & 79 \\
Total & 80 & 94 & 52 & 49 & 275 \\
\hline
\end{tabular}

Tabla 6. No. de estudiantes según estilos de aprendizaje CHAEA y Kolb

Dado que las variables utilizadas en este trabajo son nominales y no dicotómicas, para la obtención de los coeficientes de correlación de los datos obtenidos se utilizó el coeficiente Lambda de Goodman y Kruskal. Según Sierra (1989), el coeficiente Lambda se fundamenta en el carácter predictivo de los coeficientes de asociación.

Hay que tener en cuenta que estadísticamente es posible calcular Lambda haciendo caso omiso de cuál es la variable independiente y cuál es la dependiente. La fórmula para hallar lambda es la siguiente:

$$
\lambda=\frac{\sum f_{r}+\sum f_{c}-\left(F_{r}+F_{c}\right)}{2 N-\left(F_{r}+F_{c}\right)}
$$

En la que: $\Sigma$ fr es la suma de las frecuencias máximas dentro de las filas.

$\Sigma \mathrm{fc}=$ suma de las frecuencias máximas dentro de las columnas.

$\mathrm{Fr}$ = frecuencia máxima entre los totales de las filas.

$\mathrm{Fc}=$ frecuencia máxima entre los totales de las columnas.

El coeficiente Lambda obtenido para cada una de las tablas de correlación entre los estilos de aprendizaje fue:

\begin{tabular}{|c|c|}
\hline Estilos de aprendizaje & Coeficiente Lambda \\
\hline Grasha / Kolb & 0,057 \\
Grasha /CHAEA & 0,016 \\
Kolb / CHAEA & 0,046 \\
\hline
\end{tabular}

Tabla 7. Coeficiente Lamlsda de correlación entre test de estilos de aprendizaje

Como se puede observar en la tabla 7, los coeficientes de correlación obtenidos son valores muy bajos, por lo cual se puede decir que no existe una correlación significativa entre los distintos modelos de estilos de aprendizaje. Estos resultados probablemente se dan debido a los diferentes enfoques que tienen las propuestas según cada autor, como se explica a continuación:

Kolb desarrolló un modelo de aprendizaje mediante experiencias para ser aplicado en la vida adulta de las personas. Su propuesta se encamina más al nivel cognitivo de los sujetos, puesto que para Kolb, al combinarse las características del aprendizaje y la solución de problemas, y considerarlas como un proceso único, se alcanza entonces una comprensión mayor de cómo el sujeto deduce de su experiencia los conceptos, las reglas y los principios para traducir su conducta en experiencias nuevas, y de cómo adopta dichos conceptos para 
incrementar su eficacia. Para esta investigación es claro tener en cuenta que la población objeto de estudio eran estudiantes cuyas edades oscilaban entre los 14 y 19 años, por consiguiente, al aplicar esta propuesta, probablemente los estudiantes aún no tienen bien definidas las preferencias que plantea Kolb.

Por otro lado, Alonso, Gallego y Honey (1994), al basarse en la propuesta de Kolb, tienen en cuenta también varios elementos cognitivos, pero más orientados hacia la población en edad escolar, aunque también contemplan que los estilos de aprendizaje son preferencias que incluyen además de la dimensión cognitiva, la afectiva y los factores fisiológicos.

Finalmente, Grasha (1996) toma en cuenta el contexto de aprendizaje en grupos y desarrolla un modelo basado en las relaciones interpersonales que sus integrantes generan al interior de los grupos. Así, este modelo es considerado más social.

\section{Conclusiones}

De conformidad con los objetivos planteados en esta investigación, se encontró que para el caso de la población estudiada (estudiantes con modalidad en ciencias naturales), se presentó un predominio del estilo activo según el test CHAEA, estilo asimilador en el test de Kolb y estilo participativo para el test de Grasha. No obstante, como se señaló anteriormente, estos estilos no son absolutos en los estudiantes de la población, ya que tienden también a mostrar preferencias por dos, e incluso más estilos de aprendizaje, en cada uno de los modelos estudiados.
Los valores obtenidos para los coeficientes de correlación Lambda entre cada uno de los estilos de aprendizaje de los modelos estudiados son bajos, lo que mostraría que las características asociadas a cada uno de los estilos son prácticamente independientes y señalan la imposibilidad de predecir, para un sujeto cualquiera de la población, su patrón de comportamiento y estrategias de aprendizaje a partir del conocimiento de su estilo de aprendizaje en otro modelo.

Finalmente, coincidimos con la apreciación de Alonso y Gallego (2003), quienes sostienen que dada la multiplicidad de definiciones que se han trabajado sobre los estilos de aprendizaje, resulta difícil tratar de categorizar adecuadamente aquellos aspectos que resultan comunes a diferentes propuestas, sin intentar cambiar la intencionalidad de cada una de ellas. Esta dificultad se debe a que se trata de un concepto que ha sido abordado desde perspectivas diferentes, teniendo en cuenta que al existir un gran número de teorías del aprendizaje, los modelos de estilos de aprendizaje también se hacen más diversos.

\section{Referencias}

Alonso, C. M. y Gallego, D. J. (2003). Cómo diagnosticar y mejorar los estilos de aprendizaje. Madrid: UNED, Formación permanente.

Alonso, C. M., Gallego, D.J. y Honey, Y.P. (1994). Estilos de Aprendizaje. Qué son, cómo se diagnostican. Bilbao: Ediciones Mensajero.

Camargo, A. y Hederich, Ch. (2007). El estilo de enseñanza. Un concepto en búsqueda de precisión. Revista 
Pedagogía y saberes. Universidad Pedagógica Nacional. 26, pp. 31-40.

Cárdenas Oviedo, P.E. y Zapata, C.P. (2009). Estilos de enseñanza y estilos cognitivos de los docentes: Un estudio de las relaciones cognitivas, conceptuales y prácticas. Revista de Investigación 9 (1), pp. 91-103. Universidad de la Salle. Bogotá.

Clark, H. \& Kreig, R. (1988). Field dependence and strategy use. Perceptual and motor Skills, 66, 303-307.

Correa, J. (2006). Identificación de los estilos de aprendizaje en los estudiantes de fisiología del ejercicio de la Facultad de Rehabilitación y Desarrollo Humano. Revista Ciencias de la Salud, 4 (2), pp.41-53. Universidad Nacional de Colombia, Bogotá.

Dunn, R. \& Dunn, K. (1978). Teaching students through their individual learning styles: A practical approach. Nueva Jersey: EUA, Prentice Hall.

Frank, M.B. (1983). Flexibility of information processing and the memory of field independent and field dependent learners, Journal of research personality, 17, pp. 89-96.

Gardner, H. (1983). Frames of mind. The theory of the multiple intelligences. New York: Basic Books.

Grasha, A. F. (1996). Teaching with Style. A practical guide to enhancing learning by understanding teaching and learning style. Pittsburgh, PA: Alliance Publishers.

Guild, P. \& Garger, S. (1985). Marching to different drummers. Washington, D.C.: EUA. ASCD publications

Hirsh, S. y Kummerow, J. (1990). Cómo soy en realidad y cómo son los demás. Descubra su personalidad y aprove- che sus mejores cualidades. México: Paidós.

James, W.B. \& Gardner, D. L. (1995). Learning styles: Implications for distance learning. (ERIC Document Reproduction Service No. EJ 514 356).

Kolb, D. A. (1984). Experimental learning: Experience as the source of Learning and Development. New Jersey: Prentice-Hall.

Lozano, A. (2006). Estilos de aprendizaje y enseñanza. Un panorama de la estilística educativa. México: Editorial Trillas.

Mckenna, F.P. (1990). Learning implications of field dependence-independence: Cognitive style versus cognitive ability. Applied cognitive psychology, Vol 4. pp. 425-437.

Oviedo, P., Cárdenas, F., Zapata, C.P., Rendón, M., Rojas, Y., Figueroa, L. (2010) Estilos de enseñanza y estilos de aprendizaje: implicaciones para la educación por ciclos. Revista Actualidades Pedagógicas, 55, pp. 31-44. Universidad de la Salle. Bogotá.

Prado, M. (2005). Estilos de aprendizaje y rendimiento académico en entorno virtual, de estudiantes de pregrado de la Universidad Autónoma de Manizales. Revista Actas pedagógicas ,3 (11), pp. 13-18, Ibagué.

Ramírez, M. (1973) Cognitive styles and cultural democracy in education. Social science quarterly, 53, 895-904.

Revilla, D. (1998). "Estilos de aprendizaje”, Temas de Educación, Segundo Seminario Virtual del Dep. de Educación de la Pontificia Universidad Católica del Perú, Facultad y Departamento de Educación. 
Riding, R. (2002). School learning and cognitive style. London: David Fulton.

Riding, R., \& Rayner, S. (1999). Cognitive styles and learning strategies. Understanding individual differences in learning and behaviour. Londres: David Fulton Publishers.

Salkind, N.J. \& Wright, J.C. (1977). The development of reflection-impulsivity and cognitive efficiency: and integrated model. Human development, 11(20), pp. 377-387.
Sierra, R. (1989). Técnicas de investigación social, teoría y ejercicios ( $8^{a}$ ed.). España: Paraninfo S.A.

Sternberg, R. (1997). Thinking styles. Reino Unido: Cambridge University Press.

Witkin, H.A. y Goodenough, D.R. (1981) Cognitive styles: Essence and origins. New York: International University Press.

Woolfolk, A. (1995). Psicología educativa. México: Prentice Hall Hispanoamericana. 


\section{Anexo 1}

Inventario de estilo de aprendizaje (Fragmento)

(Adaptado de Kolb, 1984)

\section{Tarea}

Este cuestionario está diseñado para explorar su forma preferida de aprender

Observe las cuatro afirmaciones que aparecen en cada casilla y decida cómo ellas describen su forma de aprender. Asigne un valor de cuatro a aquella afirmación que más se aproxima a una descripción de su forma de aprender, 3 a la segunda, dos a la tercera y uno a aquella afirmación que menos describe su forma de aprender. No hay respuestas buenas o erradas.

\begin{tabular}{|c|c|c|c|c|}
\hline & $A$ & B & $\mathrm{C}$ & $\mathrm{D}$ \\
\hline I & $\begin{array}{c}\text { Me gusta } \\
\text { comprometerme }\end{array}$ & $\begin{array}{c}\text { Me gusta tomarme } \\
\text { mi tiempo antes de } \\
\text { actuar }\end{array}$ & $\begin{array}{l}\text { Soy muy particular } \\
\text { respecto a las } \\
\text { cosas que me } \\
\text { gustan }\end{array}$ & $\begin{array}{l}\text { Me gusta que las } \\
\text { cosas sean útiles }\end{array}$ \\
\hline \multicolumn{5}{|l|}{ Puntuación } \\
\hline 2 & $\begin{array}{l}\text { Me agrada ensayar } \\
\text { las cosas }\end{array}$ & $\begin{array}{l}\text { Me gusta analizar } \\
\text { las cosas y } \\
\text { descomponerlas en } \\
\text { sus partes }\end{array}$ & $\begin{array}{l}\text { Me agrada } \\
\text { probar nuevas } \\
\text { experiencias }\end{array}$ & $\begin{array}{c}\text { Me gusta mirar todos } \\
\text { los lados de las } \\
\text { situaciones }\end{array}$ \\
\hline \multicolumn{5}{|l|}{ Puntuación } \\
\hline 3 & Me gusta observar & $\begin{array}{l}\text { Me gusta analizar } \\
\text { las cosas y } \\
\text { descomponerlas en } \\
\text { sus partes }\end{array}$ & $\begin{array}{l}\text { Me agrada } \\
\text { probar nuevas } \\
\text { experiencias }\end{array}$ & $\begin{array}{l}\text { Me gusta pensar } \\
\text { acerca de las cosas }\end{array}$ \\
\hline \multicolumn{5}{|l|}{ Puntuación } \\
\hline 4 & $\begin{array}{l}\text { Acepto las personas } \\
\text { y situaciones de la } \\
\text { forma como son }\end{array}$ & $\begin{array}{l}\text { Me agrada estar } \\
\text { al tanto de lo } \\
\text { que sucede a mi } \\
\text { alrededor }\end{array}$ & Me gusta evaluar & $\begin{array}{c}\text { Me gusta asumir } \\
\text { riesgos }\end{array}$ \\
\hline \multicolumn{5}{|l|}{ Puntuación } \\
\hline 5 & $x$ & $\begin{array}{l}\text { Tengo muchas } \\
\text { preguntas }\end{array}$ & Soy lógico & $\begin{array}{l}\text { Trabajo duro para } \\
\text { conseguir las cosas }\end{array}$ \\
\hline \multicolumn{5}{|l|}{ Puntuación } \\
\hline 6 & $\begin{array}{l}\text { Me gustan las cosas } \\
\text { concretas, cosas } \\
\text { que puedo ver, } \\
\text { tocar, oler }\end{array}$ & $\begin{array}{l}\text { Me gusta estar } \\
\text { activo }\end{array}$ & Me gusta observar & $\begin{array}{c}\text { Me gustan las ideas y } \\
\text { las teorías }\end{array}$ \\
\hline \multicolumn{5}{|l|}{ Puntuación } \\
\hline 7 & $\begin{array}{l}\text { Prefiero aprender } \\
\text { aquí y ahora }\end{array}$ & $\begin{array}{c}\text { Me gusta } \\
\text { considerar y } \\
\text { reflexionar acerca } \\
\text { de las cosas }\end{array}$ & $\begin{array}{l}\text { Tiendo a pensar } \\
\text { acerca del futuro }\end{array}$ & $\begin{array}{l}\text { Me gusta ver el } \\
\text { resultado de mi } \\
\text { trabajo }\end{array}$ \\
\hline Puntuación & & & & \\
\hline
\end{tabular}




\section{Anexo 2}

Cuestionario CHAEA de estilo de aprendizaje

(Fragmento)

\section{Instrucciones para responder al cuestionario}

- Este cuestionario ha sido diseñado para identificar su estilo preferido de aprendizaje. No es un test de inteligencia, ni de personalidad.

- No hay límite de tiempo para contestar al cuestionario. No le ocupará más de 15 minutos.

- No hay respuestas correctas o erróneas. Será útil en la medida que sea sincero/a en sus respuestas.

- Si está más de acuerdo que en desacuerdo con el ítem seleccione 'Mas (+)'. Si, por el contrario, está más en desacuerdo que de acuerdo, seleccione 'Menos (-)’.

- Por favor conteste a todos los ítems.

\begin{tabular}{|c|c|c|c|}
\hline & Afirmaciones & $(+)$ & $(-)$ \\
\hline I & Tengo fama de decir lo que pienso claramente y sin rodeos. & & \\
\hline 2 & Estoy seguro de lo que es bueno y lo que es malo, lo que está bien y lo que está mal. & & \\
\hline 3 & Muchas veces actúo sin mirar las consecuencias. & & \\
\hline 4 & Normalmente trato de resolver los problemas metódicamente y paso a paso. & & \\
\hline 5 & Creo que los formalismos coartan y limitan la actuación libre de las personas. & & \\
\hline 6 & $\begin{array}{l}\text { Me interesa saber cuáles son los sistemas de valores de los demás y con qué criterios } \\
\text { actúan. }\end{array}$ & & \\
\hline 7 & $\begin{array}{l}\text { Pienso que el actuar intuitivamente puede ser siempre tan válido como actuar } \\
\text { reflexivamente. }\end{array}$ & & \\
\hline 8 & Creo que lo más importante es que las cosas funcionen. & & \\
\hline 9 & Procuro estar al tanto de lo que ocurre aquí y ahora. & & \\
\hline
\end{tabular}




\title{
Anexo 3
}

\author{
Cuestionario Grasha-Riechmann \\ Escala de estilo de aprendizaje de los estudiantes \\ (Forma general) \\ (Fragmento)
}

El siguiente cuestionario ha sido diseño para ayudarlo a clarificar sus actitudes y sentimientos hacia los cursos que Ud. toma en la actualidad en el colegio. No hay respuesta buena o errada para cada cuestión. Responda a los ítems que aparecen en el listado de abajo siguiendo la escala que se señala a continuación. Siga las instrucciones de la persona que le aplica el cuestionario.

\begin{tabular}{|l|}
\hline Use una puntuación de I si está fuertemente en desacuerdo con la afirmación \\
\hline Use una puntuación de 2 si está moderadamente en desacuerdo con la afirmación \\
\hline Use una puntuación de 3 si está indeciso \\
\hline Use una puntuación de 4 si está moderadamente de acuerdo con la afirmación \\
\hline Use una puntuación de 5 si está fuertemente de acuerdo con la afirmación \\
\hline
\end{tabular}

\begin{tabular}{|c|l|l|}
\hline I & Prefiero trabajar individualmente en las tareas de mis cursos & \\
\hline 2 & Frecuentemente me aburro durante las clases & \\
\hline 3 & $\begin{array}{l}\text { Trabajar con otros estudiantes las actividades de clase es algo que } \\
\text { disfruto }\end{array}$ & \\
\hline 4 & $\begin{array}{l}\text { Me gusta que los profesores digan exactamente qué esperan de los } \\
\text { estudiantes }\end{array}$ & \\
\hline 5 & $\begin{array}{l}\text { Para sobresalir, es necesario competir con otros estudiantes por la } \\
\text { atención del profesor }\end{array}$ & \\
\hline 6 & Hago lo que se me pida para aprender el contenido de mis clases & \\
\hline 7 & $\begin{array}{l}\text { Mis ideas acerca del contenido con frecuencia son tan buenas como } \\
\text { aquellas de los libros de texto }\end{array}$ & \\
\hline 8 & Las actividades de clase son usualmente aburridas & \\
\hline
\end{tabular}

